\title{
Anwendungsmöglichkeiten der optischen Kohärenztomo- graphie beim trockenen Auge
}

\author{
Andreas Schlatter · Cornelia Hirn · Stefan Palkovits · René M. Werkmeister · Oliver Findl • Gerhard Garhöfer • \\ Doreen Schmidl
}

Eingegangen: 2. März 2021 / Angenommen: 22. April 2021 / Online publiziert: 1. Juni 2021

(C) Der/die Autor(en) 2021

\begin{abstract}
Zusammenfassung Durch die stetige Weiterentwicklung und Verbesserung der Technik hat die optische Kohärenztomographie (OCT) in den letzten Jahren neue Möglichkeiten zur Beurteilung der Strukturen des vorderen Augenabschnittes eröffnet. Aufgrund der Darstellung kleinster Strukturen, wie beispielsweise des Tränenfilms, nimmt die OCT in der Diagnostik und Verlaufsbeurteilung des trockenen Auges eine immer wichtigere Rolle ein. Der vorliegende Beitrag gibt einen Überblick über die derzeitigen Einsatzmöglichkeiten der OCT beim trockenen Auge.
\end{abstract}

Schlüsselwörter Optische Kohärenztomographie Trockenes Auge · Augenoberflächenerkrankung · Tränenfilm $\cdot$ Meibom-Drüsen

\section{Application possibilities of optical coherence tomography in dry eye disease}

Summary Due to the continuous development and improvement of the technology, optical coherence tomography (OCT) has yielded new approaches for the assessment of structures of the anterior segment of

\author{
A. Schlatter · G. Garhöfer . \\ Assoc.-Prof. PD Dr. D. Schmidl, PhD ( $₫)$ \\ Universitätsklinik für Klinische Pharmakologie, \\ Medizinische Universität Wien, Währinger Gürtel \\ 18-20, 1090 Wien, Österreich \\ doreen.schmidl@meduniwien.ac.at
}

A. Schlatter · C. Hirn · S. Palkovits · O. Findl

Augenabteilung, Hanusch-Krankenhaus, Vienna

Institute for Research in Ocular Surgery (VIROS),

Heinrich-Collin-Str. 30, 1140 Wien, Österreich

\section{R. M. Werkmeister}

Zentrum für Medizinische Physik und Biomedizinische Technik, Medizinische Universität Wien, Währinger Gürtel 18-20, 1090 Wien, Österreich the eye in recent years. By enabling imaging of small structures, such as the tear film, the role of OCT in the diagnostics and follow-up of dry eye disease is constantly increasing. The present article provides an overview about current application possibilities of OCT in dry eye disease.

Keywords Optical coherence tomography $\cdot$ Dry eye disease - Ocular surface disease - Tear film . Meibomian glands

Die optische Kohärenztomographie (OCT) stellt die wahrscheinlich größte technische Innovation in der ophthalmologischen Praxis der letzten Jahrzehnte dar und ist im modernen klinischen Alltag für die Diagnostik und die Verlaufsbeurteilung verschiedenster Erkrankungen kaum mehr wegzudenken. Das Funktionsprinzip der OCT wurde erstmals im Jahr 1991 von Huang et al. anhand von In-vitro-OCT-Scans in der Fachzeitschrift Science beschrieben [1]. Die Autoren wiesen bereits damals auf die hohe klinische Relevanz des neu entwickelten Systems für die Diagnostik von verschiedenen Augenerkrankungen, wie beispielsweise Glaukom, altersabhängiger Makuladegeneration (AMD) oder Makulaödem, hin.

Nach stetiger Verbesserung sowohl der Aufnahmegeschwindigkeit als auch der Auflösung der Geräte findet die OCT in der modernen Medizin ein breites Anwendungsgebiet, welches sich nicht nur auf die Augenheilkunde beschränkt. Jedoch hat sich im Laufe der Zeit auch die Anzahl der ophthalmologischen Anwendungsbereiche deutlich erweitert. Im Jahr 1994 publizierten Izatt et al. das erste System zur Darstellung des Vorderabschnittes, mit welchem die Hornhaut, die Vorderkammer und die Linse dargestellt werden konnten [2]. 
Die so ermöglichte Schnittbilddarstellung des vorderen Augenabschnittes bietet große Vorteile beispielsweise in der nichtinvasiven Beurteilung kornealer Strukturen [3], des Kammerwinkels [4] oder auch in der Biometrie im Rahmen von Kataraktoperationen [5]. Mit der immer höher werdenden axialen Auflösung ist es in den letzten Jahren gelungen, auch Strukturen des Vorderabschnittes im Bereich von wenigen Mikrometern, wie beispielsweise den Tränenfilm, darzustellen [3, 6-8].

Bisher etablierte Untersuchungsmethoden in der Routinediagnostik von Augenoberflächenerkrankungen, zu welchen unter anderem das Syndrom des trockenen Auges (= Keratoconjunctivitis sicca [KCS]), die Meibom-Drüsen-Dysfunktion, Blepharitis und allergische Konjunktivitis zählen [9], sind sehr häufig abhängig von der Beurteilung durch den Untersucher und damit nicht vollständig objektivierbar. Zudem mangelt es den konventionellen Untersuchungsmethoden oft an zufriedenstellender Reproduzierbarkeit [10]. Die oftmals große Diskrepanz zwischen dem klinisch beschriebenen Schweregrad der Erkrankung und den subjektiven Patientenbeschwerden sowie Invasivität der Untersuchungsmethoden selbst spielen zudem eine limitierende Rolle [11].

Aufgrund der fortschreitenden Digitalisierung im beruflichen und privaten Alltag und der damit verbundenen vermehrten Bildschirmarbeit hat die Inzidenz von Oberflächenerkrankungen des Auges, v.a. der KCS, stetig zugenommen [12, 13]. Die OCT hat aufgrund der Objektivität und der guten Reproduzierbarkeit das Potenzial, in der Diagnostik und Verlaufsbeurteilung in Zukunft eine wichtige Rolle einzunehmen.

\section{Technische Aspekte der OCT-Untersuchung der Augenoberfläche}

Grundsätzlich wird bei der OCT ein Lichtstrahl mit niedriger Kohärenzlänge in einem interferometrischen Aufbau auf einen Strahlteiler gerichtet. Von dort aus wird er in 2 verschiedene Arme aufgeteilt, einen Referenzarm und einen Probenarm. Im Referenzarm wird der Lichtstrahl von einem Spiegel, im Probenarm von den untersuchten Gewebeschichten gestreut und reflektiert. Das in beiden Armen reflektierte Licht trifft sich wieder im mittigen Strahlteiler und wird von dort aus auf einen Detektor gerichtet. Anhand der dort detektierten Interferenzmuster lässt sich ein A-Scan, vergleichbar mit jenem der Sonographie, errechnen, welcher durch die optischen Eigenschaften und unterschiedlichen Brechungsindizes des untersuchten Gewebes charakterisiert wird [3]. Aus der Zusammensetzung vieler durch laterales Verschieben des Messstrahls über das Gewebe gewonnener benachbarter A-Scans lässt sich wiederum ein sog. B-Scan, welcher einem Querschnitt durch das Gewebe entspricht, erstellen. Im vorderen Augenab- schnitt kommen hauptsächlich Fourier-domain(FD)Systeme zum Einsatz.

Rein technisch betrachtet, unterscheidet man in der OCT die axiale und die transversale Auflösung. Die axiale Auflösung ist primär abhängig von der Kohärenzlänge der verwendeten Lichtquelle. Diese Kohärenzlänge ist wiederum direkt abhängig von der Bandbreite und der zentralen Wellenlänge dieser. Damit nimmt die Charakteristik der verwendeten Lichtquelle, meist ein Breitbandlaser oder eine Superlumineszenzdiode, eine zentrale Rolle für die Bildgebungseigenschaften von OCT-Systemen zur Betrachtung von Augenoberflächenerkrankungen ein [14]. Die transversale Auflösung ist im Gegensatz zur Mikroskopie entkoppelt von der axialen Auflösung und hauptsächlich abhängig von der Strahlgeometrie und der Brennweite der verwendeten Linsen zur Fokussierung des Messstrahls [3, 15].

Für die Bildgebung des vorderen Augenabschnittes und der darstellbaren morphologischen Details nimmt die axiale Auflösung der Systeme eine zentrale Rolle ein, da die zu untersuchenden Gewebeschichten - insbesondere die Hornhaut und die Linse im rechten Winkel zur Scanebene angeordnet sind und für die verschiedenen Untersuchungen v.a. die Vermessung der Strukturen in axialer Ebene wichtig sind.

Kommerzielle Vorderabschnitts-OCT-Systeme erreichen eine axiale Auflösung von ca. 5-15 $\mu \mathrm{m}$. Diese Auflösung ist zu gering, um den ca. 3-5 $\mu$ m dicken Tränenfilm darzustellen. Um den Tränenfilm bildlich aufzulösen und klinisch signifikante Änderungen der Tränenfilmdicke zu detektieren, sind axiale Auflösungen von ca. $1 \mu \mathrm{m}$ oder weniger notwendig [16]. Inzwischen wurden jedoch Prototypen ultrahochauflösender OCT-Systeme vorgestellt, welche eine entsprechende Auflösung erreichen und damit den Tränenfilm visualisieren und vermessen können [17, 18].

Gerade die stetige Verbesserung der verwendeten Lichtquellen, der Scan-Geschwindigkeiten sowie der Analysemethoden haben in den letzten Jahren zu erheblichen Fortschritten in der Qualität der OCT-Bilddaten und damit zu erweiterten diagnostischen Möglichkeiten im vorderen Augensegment und der Augenoberfläche geführt.

\section{Anwendungen der OCT in der Diagnostik von Augenoberflächenerkrankungen}

\section{Tränenfilm}

Der präkorneale Tränenfilm spielt eine zentrale Rolle bei der Entstehung der KCS, wie auch in der Definition der Erkrankung im Dry Eye Workshop 2017 hervorgehoben wird [19].

Bisher basierte die Beurteilung des Tränenfilms hauptsächlich in der Überprüfung der Stabilität (via Messung der Tränenfilmaufreißzeit) oder der Pro- 


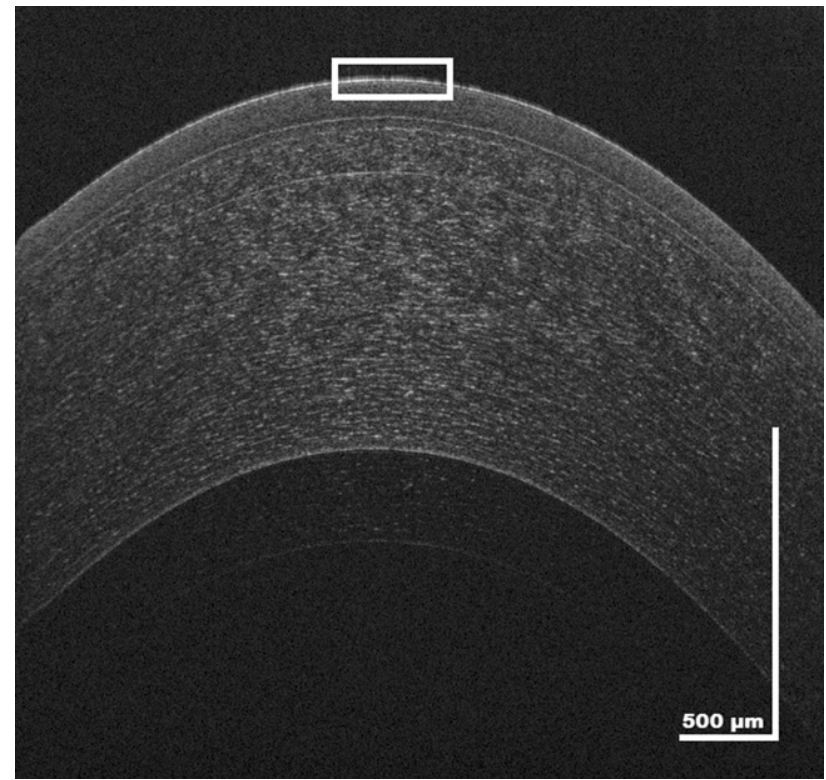

Abb. 1 Horizontaler, zentraler ultrahochauflösender (engl. „ultra-high resolution“ [UHR]) OCT-Scan der Hornhaut. Der dem Hornhautepithel aufliegende Tränenfilm ist ungefähr 3-5 $\mu \mathrm{m}$ dick und ist durch hyperdense Reflektivität gekennzeichnet (zentral markiert durch ein weißes Rechteck)

duktion (via Schirmer-Test) [20]. Der Tränenfilm setzt sich aus verschiedenen Komponenten zusammen und dient der Ernährung und dem Schutz der Hornhaut. Darüber hinaus besitzt er als Grenzfläche zwischen Luft und Auge aufgrund der unterschiedlichen Brechungsindizes wichtige refraktive Eigenschaften [21, 22].

Die äußere Lipidschicht wird von den sich in den Augenlidern befindlichen Meibom-Drüsen produziert und in den Tränenfilm sezerniert. Sie dient als hydrophobe Barriere und wirkt damit dem Verdunsten der wässrigen Phase, welche den größten Anteil des Tränenfilms ausmacht, entgegen. Eine Verringerung des lipidhaltigen Anteils des Tränenfilms resultiert in einer verringerten Stabilität der wässrigen Phase auf der Augenoberfläche und erhöht damit das Risiko, eine evaporative Form der KCS zu entwickeln. Diese Form der KCS wird häufig durch eine Meibom-Drüsen-Dysfunktion verursacht [23].

Die wässrige Phase wird von den Tränendrüsen sowie den akzessorischen Krause- und Wolfring-Drüsen produziert. Eine Unterfunktion dieser verursacht die hypovolämische Form der KCS (= ADDE - „aqueous deficiency dry eye“), bei welcher durch pathologische Veränderungen der Tränendrüse, wie beispielsweise beim sog. Sjögren-Syndrom [24] vorkommend, die Produktion der wässrigen Tränenbestandteile vermindert ist.

Die innere Muzinschicht wird vorwiegend von den in der Bindehaut vorkommenden Becherzellen produziert. Sie wird als raue Grenzschicht zwischen Hornhautepithel und wässriger Phase beschrieben [25]. Ein Verlust dieser Becherzellen gilt als charak- teristisch für viele Augenoberflächenerkrankungen und ist beispielsweise mittels Impressionszytologie nachweisbar [26].

Durch die stetige Verbesserung der axialen Auflösung von OCT-Prototypen ist es nun möglich, den Tränenfilm visuell darzustellen und quantitativ zu vermessen (Abb. 1; [3, 17, 25, 27]). Bei Gesunden wurde eine Tränenfilmdicke („tear film thickness“ [TFT]) von ungefähr 3-5 $\mu \mathrm{m}$ mittels OCT gemessen, während sie bei PatientInnen mit KCS reduziert zu sein scheint [18, 28-32].

Auch zeigt die Methode der Messung der TFT mittels OCT eine sehr hohe Reproduzierbarkeit und Wiederholbarkeit [18]. Des Weiteren konnte gezeigt werden, dass die TFT negativ mit dem Ocular Surface Disease Index (OSDI), einem Symptomfragebogen, korreliert und es auch einen signifikanten Zusammenhang zwischen TFT und Schirmer-Test bzw. Tränenfilmaufreißzeit gibt $[6,33]$.

Dies deutet darauf hin, dass sich die TFT als zukünftiger Biomarker für die Diagnose und Verlaufsbeobachtung der KCS eignet. Dies zeigt sich auch darin, dass eine verminderte TFT durch Therapie erhöht werden kann. So konnte im Rahmen zahlreicher Studien gezeigt werden, dass Eintropfen von verschiedenen Lubrikanzien zu einer signifikanten Zunahme der TFT führt [28-30, 32, 34, 35]. Des Weiteren kann diese Technik auch dazu verwendet werden, die Verweilzeit von Lubrikanzien auf der Hornhautoberfläche zu bestimmen. Diese bewegt sich je nach chemischer Zusammensetzung und Viskosität der verwendeten Substanzen zwischen einigen Minuten und mehreren Stunden [31].

Neben der Verweilzeit auf der Augenoberfläche nach einmaliger Applikation besteht natürlich auch erhebliches Interesse daran, Informationen über den Effekt einer kontinuierlichen Therapie auf die TFT $\mathrm{zu}$ erhalten. So konnte gezeigt werden, dass eine 4-wöchige Therapie mit Perfluorohexyloctane-Augentropfen im Laufe der Behandlung zu einer kontinuierlichen Zunahme der TFT führt [36]. Auch unter Therapie mit Hydrocortison-Augentropfen und sogar über die Therapiedauer verbesserte sich die TFT über die Zeit [37]. Diese Daten zeigen, dass die Vermessung des Tränenfilms mittels OCT eine zukünftige Methode darstellen könnte, den klinischen Verlauf in PatientInnen mit trockenem Auge zu verfolgen.

Während die oben genannte quantitative Vermessung der TFT nur Aussagen zur Gesamtdicke liefert, befinden sich derzeit auch Verfahren zur Vermessung einzelner Schichten in Entwicklung. So gibt es schon Erfolg versprechende Ansätze zur Untersuchung der Lipidschichtdicke und der wässrigen Phase mittels OCT [25, 38, 39].

Neben der quantitativen Vermessung des Tränenfilms zu einem bestimmten Zeitpunkt wurden auch Ansätze entwickelt, um Aussagen über das dynamische Verhalten des Tränenfilms treffen zu können, indem in kurzen Intervallen Volumina vermessen 


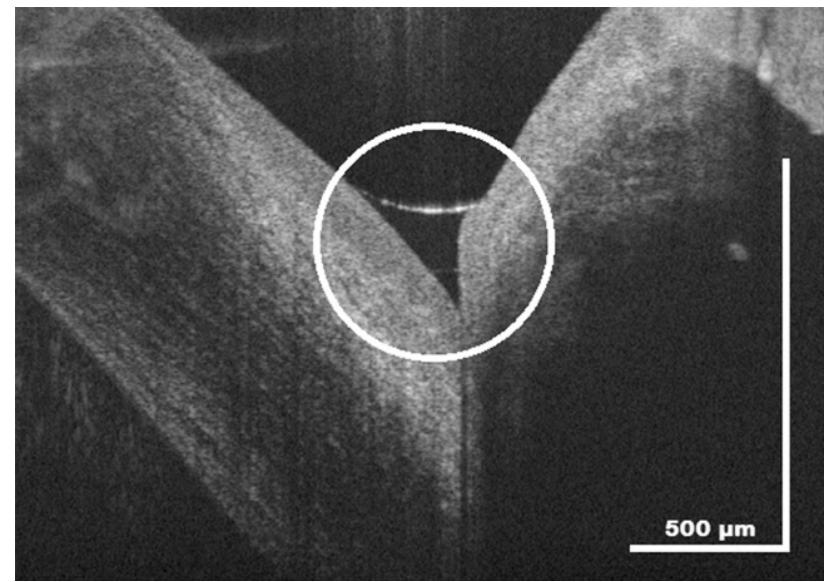

Abb. 2 Vertikaler, zentraler UHR-OCT-Scan am Übergang zwischen Hornhaut (links) und Lidkante (rechts). Der Tränenfilmmeniskus befindet sich in der Mitte des Scans (weiß eingekreist) genau am Übergang von Hornhaut und Lidkante und ermöglicht eine Schätzung der vorhandenen Tränenflüssigkeit

werden $[27,40]$. Durch volumetrische Scans der Augenoberfläche und En-face-Projektionen der Daten ist es möglich, äquivalent zur Tränenfilmaufreißzeit (TFBUT) das Aufreißverhalten des Tränenfilms zu beurteilen, jedoch ist dies zur Zeit aufgrund der Tatsache, dass nur ein kleiner Teil der Augenoberfläche gescannt werden kann, nur eingeschränkt möglich [3, $18]$.

\section{Tränenfilmmeniskus}

Der Großteil der sich auf der Augenoberfläche befindlichen Tränenflüssigkeit (ca. 75-90\%) sammelt sich in den Tränenfilmmenisken, welche sich am Übergang von oberen bzw. unteren Augenlidern und Augenoberfläche befinden, an [41-44]. Obwohl eine Beurteilung des Tränenmeniskus auch an der Spaltlampe möglich ist, bietet die Untersuchung mittels OCT zahlreiche Vorteile, da es hierbei zu keinem Reflextränenfluss aufgrund der hellen Beleuchtung kommt und die Vermessung mit hoher Reproduzierbarkeit erfolgen kann (Abb. 2; [45, 46]).

Da die Messung des Tränenfilmmeniskus eine weniger hohe axiale Auflösung (ca. $10 \mu \mathrm{m}$ ) als die Messung der TFT erfordert und damit mit kommerziellen OCT-Systemen möglich ist [3], wurde in den letzten Jahren eine Vielzahl an klinischen Studien zum Tränenfilmmeniskus durchgeführt. Es konnte, ähnlich wie für die TFT, gezeigt werden, dass Tränenmeniskusparameter wie Höhe, Fläche, Volumen, Tiefe und Radius sehr gut mit „klassischen“ Untersuchungen, z.B. OSDI, Fluoreszeinfärbung der Hornhaut oder Schirmer Test korrelieren [39, 47-50]. Auch kann mithilfe der verschiedenen genannten Parameter zwischen verschiedenen Formen der KCS unterschieden werden, was in Zukunft hilfreich bei der Differenzialdiagnose sein könnte [51].
Analog zur TFT kommt es auch zu einem Anstieg der Tränenmeniskusparameter nach 1-maliger Verabreichung von topischen Lubrikanzien [52-54]. Auch scheint sich die Methode gut zur Therapieevaluierung über einen längeren Zeitraum zu eignen, z. B. konnte gezeigt werden, dass topische Therapie mit Cyclosporin über 2 Monate zu einem Anstieg der Höhe und des Volumens des Tränenmeniskus führt [44].

\section{Meibom-Drüsen}

Da durch die OCT auch die Darstellung intransparenter Medien möglich ist, lassen sich bei entsprechender Eindringtiefe auch die im Ober- bzw. Unterlid befindlichen Meibom-Drüsen visualisieren und pathologische Veränderungen detektieren [55-59]. Dies ermöglicht im Gegensatz zur häufig verwendeten Infrarotmeibographie eine 3-dimensionale Darstellung der Drüsen, bietet jedoch einen kleineren gescannten Bereich, welcher je nach OCT-System einen Bereich von wenigen Quadratmillimetern abdeckt. Die Infrarotmeibographie erlaubt zwar die Begutachtung ausgefallener Drüsen als Ganzes, jedoch ist keine detaillierte Bewertung der Morphologie der Azini und der Ausführungsgänge möglich. Dies wiederum ermöglichen verschiedene vorgestellte OCT-Systeme. So konnte beispielsweise die morphologische Veränderung der Azini der Meibom-Drüsen bei PatientInnen mit Syndrom des trockenen Auges nachgewiesen werden [55]. Da die Azini die sekretorische Komponente der Meibom-Drüsen bilden, könnte die OCT in Zukunft eine wichtige Rolle in der Diagnostik und Therapieentscheidung bei PatientInnen mit MeibomDrüsen-Dysfunktion spielen und damit eine wichtige Ergänzung zur Infrarotmeibographie darstellen. Auch die Auswirkungen einer durchgeführten Lidrandmassage auf die Drüsenöffnungen am Lidrand konnten beispielsweise mittels OCT gezeigt werden [57].

\section{LIPCOF}

Die sog. Lid-parallelen konjunktivalen Falten (engl. „lid-parallel conjunctival folds“ [LIPCOF]) finden sich v. a. bei PatientInnen mit Syndrom des trockenen Auges am unteren temporalen Lidwinkel und entstehen wahrscheinlich durch erhöhte Friktion zwischen der palpebralen und der bulbären Konjunktiva bei mangelnder Benetzung der Augenoberfläche. Sie werden in der Regel im Rahmen der klinischen Untersuchung am Spaltlampenmikroskop beobachtet. Im Gegensatz dazu bietet die OCT jedoch den Vorteil, eine objektivere und genauere Aussage über Größe und Anzahl der LIPCOF zu treffen [60]. Vor allem bei Kontaktlinsenträgern lassen sich die LIPCOF durch die OCT äußerst präzise bewerten [61]. Die LIPCOF können jedoch auch die Vermessung des Tränenfilmmeniskus beeinflussen, da sie diesen durch ihre Lage verdrängen können [62, 63]. Die Untersuchung von LIPCOFs 
mit OCT spielt bislang im Vergleich zu anderen Methoden jedoch eine untergeordnete Rolle.

\section{Tränendrüse und Punctum lacrimale}

Bisher gibt es kaum Daten über die Anwendung der OCT, um in vivo die Morphologie der Tränendrüsen genauer zu analysieren. Doh et al. stellten im Jahr 2015 in einem Report dieses Einsatzgebiet der OCT vor [64]. Für eine Darstellung der Tränendrüse muss das Oberlid sehr stark hinaufgezogen werden, damit diese frei sichtbar ist. Es lassen sich dadurch die Strukturen der Glandula lacrimalis in Schnittbilddarstellung betrachten. Die Autoren des Reports konnten auf den OCTScans Parenchym, Azini, interlobuläre Gänge, intralobuläre Gänge und größere Ausführungsgänge beobachten. Bisher gibt es jedoch keine weiterführenden Studien zur OCT-Analyse der Tränendrüse.

Auch die Öffnungen der Punctum lacrimalia lassen sich mittels Vorderabschnitts-OCT hervorragend darstellen. Diese Technik ist bisher jedoch ebenfalls wenig etabliert und lässt aufgrund der geringen Eindringtiefe der OCT nur Aussagen über die Öffnung zu, nicht jedoch über die Durchgängigkeit des kompletten Ductus nasolacrimalis [65-69].

\section{OCT-Angiographie des vorderen Augenabschnitts}

Vor allem in der Beurteilung von retinalen Erkrankungen gewann die funktionelle Erweiterung der OCT, die OCT-Angiographie (OCT-A), in den letzten Jahren immer mehr an Bedeutung [70]. Diese Technik erlaubt eine nichtinvasive funktionelle Darstellung des Gefäßsystems der obersten Schichten des untersuchten Gewebes. Die Technik basiert auf der zeitlichen Änderung des OCT-Signals an einer bestimmten Stelle, hervorgerufen durch die Bewegung des Blutes, insbesondere der Erythrozyten, in den Gefäßen. Somit kann stationäres Gewebe (keine zeitliche Änderung) von Blutfluss (zeitliche Änderung) unterschieden werden [71]. Diese Technik kann auch in den Gewebeschichten des vorderen Augenabschnittes angewandt werden. In den letzten Jahren wurden vereinzelt Studien zur OCT-A-Anwendung im vorderen Augensegment durchgeführt, z.B. bei GlaukompatientInnen oder PatientInnen mit entzündlichen Erkrankungen [72, 73]. Des Weiteren konnten die Vorteile der Anwendung von OCT-A in der Beurteilung der limbalen Gefäße gegenüber der klinischen Untersuchung nach Unfällen mit chemischen Substanzen demonstriert werden [74]. Möglicherweise kann diese Technologie auch in der Zukunft bei PatientInnen mit KCS zur Anwendung kommen, ein mögliches Einsatzgebiet wäre beispielsweise die objektive Beurteilung von Bindehauthyperämien, welche derzeit nur mittels Vergleich mit standardisierten Fotografien durchgeführt wird [75].

\section{Diskussion}

Sowohl die OCT als auch die OCT-A nehmen eine immer größer werdende Rolle in der Beurteilung des vorderen Augenabschnittes ein. In den letzten Jahren wurden in zahlreichen klinischen Studien neue Anwendungsbereiche bei Augenoberflächenerkrankungen vorgestellt. Diese Anwendungen beschränken sich bisweilen größtenteils auf wissenschaftliche Forschungsprojekte mit experimentellen OCT-Systemen $[17,18]$. Es ist jedoch anzunehmen, dass sowohl die OCT als auch die OCT-A bei Augenoberflächenerkrankungen in den kommenden Jahren auch in der klinischen Praxis breitere Anwendung finden werden. Mit den immer besser werdenden Bildparametern könnte die OCT zu anderen Verfahren, wie beispielsweise die Darstellung kornealer Nerven mittels Konfokalmikroskopie oder die Darstellung von MeibomDrüsen mittels Infrarotmeibographie, eine wertvolle Alternative darstellen [43].

Vor allem in der Diagnostik, Verlaufsbeurteilung und Therapieentscheidung des Syndroms des trockenen Auges könnte die ultrahochauflösende OCT mit objektiven Messparametern wie Tränenfilmdicke, Tränenfilmmeniskus und En-face-Tränenfilmaufreißzeit eine hilfreiche Erweiterung zu den bisher etablierten und oftmalig vom Untersucher abhängigen Untersuchungsmethoden darstellen. Neben den vielversprechenden Anwendungen im Bereich der Vermessung des Tränenfilms könnte die ultrahochauflösende OCT auch bei anderen Augenoberflächenerkrankungen weitere diagnostische Möglichkeiten eröffnen. So stellen beispielweise die hochauflösende Darstellung der Meibom-Drüsen bzw. des Lidrandes interessante Ansätze für deren Beurteilung dar [55-59, 65-69].

Die OCT bietet in der Beurteilung des vorderen Augenabschnittes einige Vorteile: Vor allem die kurze Dauer der Messungen von wenigen Sekunden und die Nichtinvasivität erweisen sich im Vergleich mit anderen Untersuchungsmethoden, wie beispielweise dem 5-minütigen invasiven Schirmer-Test, als vergleichsweise angenehm für die PatientInnen. Zudem ermöglicht die OCT dem Untersucher die Beurteilung der Morphologie verschiedener Gewebestrukturen in vivo und in Echtzeit.

Nachteile der OCT sind die noch derzeit hohen Kosten, welche zu einem Großteil auf die aufwendigen Lichtquellen zurückfallen, die geringe Eindringtiefe in das Gewebe und die erforderliche Mitarbeit der PatientInnen. Gerade bei der Vermessung des Tränenfilms, welche in der Regel einige Sekunden dauert, können vorzeitiges oder verstärktes Blinzeln, unzureichende Fixation, aber auch kleinste unwillkürliche Augenbewegungen wie beispielsweise Sakkaden die Messung erschweren. Deshalb ist eine adäquate Instruktion der PatientInnen unverzichtbar für den Erfolg der Messung. 
Der Aspekt der geringen Eindringtiefe ist im vorderen Augenabschnitt vernachlässigbar, da Strukturen wie beispielsweise die Hornhaut und der Tränenfilm transparent sind. Auch der künftige Einsatz der OCT-A im vorderen Augensegment bietet vielversprechende Ansätze, sollte jedoch durch weitere klinische Studien noch weiter evaluiert werden $[72,73,76]$.

Alles in allem gibt es neben den bereits etablierten Anwendungsgebieten der OCT im hinteren Augenabschnitt einige vielversprechende Ansätze in der Diagnostik von Augenoberflächenerkrankungen durch spezielle OCT-Systeme zur Darstellung des vorderen Augenabschnitts mit seinen speziellen Anforderungen an das Messsystem. Gerade der Einsatz der ultrahochauflösenden OCT in der Tränenfilmdiagnostik hat sich in einigen klinischen Studien als valide und klinisch relevant erwiesen und könnte in Zukunft auch in der klinischen Routine eine wertvolle Ergänzung in der Beurteilung von Augenoberflächenerkrankungen darstellen.

Funding Open access funding provided by Medical University of Vienna.

Interessenkonflikt A. Schlatter, C. Hirn, S. Palkovits, R.M. Werkmeister, O. Findl, G. Garhöfer und D. Schmidl geben an, dass kein Interessenkonflikt besteht.

Open Access Dieser Artikel wird unter der Creative Commons Namensnennung 4.0 International Lizenz veröffentlicht, welche die Nutzung, Vervielfältigung, Bearbeitung, Verbreitung und Wiedergabe in jeglichem Medium und Format erlaubt, sofern Sie den/die ursprünglichen Autor(en) und die Quelle ordnungsgemäß nennen, einen Link zur Creative Commons Lizenz beifügen und angeben, ob Änderungen vorgenommen wurden.

Die in diesem Artikel enthaltenen Bilder und sonstiges Drittmaterial unterliegen ebenfalls der genannten Creative Commons Lizenz, sofern sich aus der Abbildungslegende nichts anderes ergibt. Sofern das betreffende Material nicht unter der genannten Creative Commons Lizenz steht und die betreffende Handlung nicht nach gesetzlichen Vorschriften erlaubt ist, ist für die oben aufgeführten Weiterverwendungen des Materials die Einwilligung des jeweiligen Rechteinhabers einzuholen.

Weitere Details zur Lizenz entnehmen Sie bitte der Lizenzinformation auf http://creativecommons.org/licenses/by/4. $0 /$ deed.de.

\section{Literatur}

1. Huang D, Swanson EA, Lin CP, Schuman JS, Stinson WG, Chang W, et al. Optical coherence tomography. Science. 1991;254(5035):1178-81.

2. IzattJA, Hee MR, Swanson EA, Lin CP, Huang D, Schuman JS, et al. Micrometer-scale resolution imaging of the anterior eye in vivo with optical coherence tomography. Arch Ophthalmol. 1994;112(12):1584-9.

3. Ang M, Baskaran M, Werkmeister RM, Chua J, Schmidl D, Aranha dos Santos V, et al. Anterior segment optical coherence tomography. Prog Retin Eye Res. 2018;66:132-56.

4. Triolo G, Barboni P, Savini G, De Gaetano F, Monaco G, David $A$, et al. The use of anterior-segment optical-coherence tomography for the assessment of the Iridocorneal angle and its alterations: update and current evidence. J Clin Med. 2021;10(2):231.

5. Nguyen P, Chopra V. Applications of optical coherence tomography in cataract surgery. Curr Opin Ophthalmol. 2013;24(1):47-52.

6. Garhöfer G, Aranha dos Santos V, Stegmann H, Schmidl D, Adzhemian N, Werkmeister RM, et al. The association between tear film thickness as measured with OCT and symptoms and signs of dry eye disease: a pooled analysis of 6 clinical trials. J Clin Med.2020;9(11):3791.

7. Kottaiyan R, Yoon G, Wang Q, Yadav R, Zavislan JM, Aquavella JV. Integrated multimodal metrology for objective and noninvasive tear evaluation. Ocul Surf. 2012;10(1):43-50.

8. Huang J, Yuan Q, Zhang B, Xu K, Tankam P, Clarkson E, et al. Measurement of a multi-layered tear film phantom using optical coherence tomography and statistical decision theory. Biomed OptExpress. 2014;5(12):4374-86.

9. Khanna RC. Ocular surface disorders. Community Eye Health. 2017;30(99):S1-S2.

10. Han SB, Liu YC, Mohamed-Noriega K, Tong L, Mehta JS. Objective imaging diagnostics for dry eye disease. J Ophthalmol. 2020; https://doi.org/10.1155/2020/3509064.

11. Ong ES, Felix ER, Levitt RC, Feuer WJ, Sarantopoulos CD, Galor A. Epidemiology of discordance between symptoms and signs of dry eye. BrJOphthalmol. 2018;102(5):674-9.

12. Moon JH, Kim KW, Moon NJ. Smartphone use is a riskfactor for pediatric dry eye disease according to region and age: a case control study. BMCOphthalmol.2016;16(1):188.

13. Rossi GCM, Scudeller L, Bettio F, Pasinetti GM, Bianchi PE. Prevalence of dry eye in video display terminal users: a cross-sectional Caucasian study in Italy. Int Ophthalmol. 2019;39(6):1315-22.

14. Drexler W, Morgner U, Ghanta RK, Kärtner FX, Schuman JS, Fujimoto JG. Ultrahigh-resolution ophthalmic optical coherence tomography. NatMed.2001;7(4):502-7.

15. Kostanyan T, Wollstein G, Schuman JS. New developments in optical coherence tomography. Curr Opin Ophthalmol. 2015;26(2):110-5.

16. Bai Y, Nichols JJ. Advances in thickness measurements and dynamic visualization of the tear film using non-invasive optical approaches. Prog Retin Eye Res. 2017;58:28-44.

17. Yadav R, Lee K-S, Rolland JP, Zavislan JM, Aquavella JV, Yoon G. Micrometer axial resolution OCT for corneal imaging. Biomed OptExpress. 2011;2(11):3037-46.

18. Werkmeister RM, Alex A, Kaya S, Unterhuber A, Hofer B, Riedl J, et al. Measurement of tear film thickness using ultrahigh-resolution optical coherence tomography. Invest Ophthalmol Vis Sci. 2013;54(8):5578-83.

19. Craig JP, Nichols KK, Akpek EK, Caffery B, Dua HS, Joo CK, et al. TFOS DEWS II definition and classification report. Ocul Surf. 2017;15(3):276-83.

20. Wolffsohn JS, Arita R, Chalmers R, Djalilian A, Dogru M, Dumbleton K, et al. TFOS DEWS II diagnostic methodology report. OculSurf. 2017;15(3):539-74.

21. KohS. Mechanisms of visual disturbance in dry eye. Cornea. 2016;35(Suppl 1):S83-S8.

22. KohS, TungCI, InoueY, Jhanji V.Effects of tearfilm dynamics on quality of vision. BrJ Ophthalmol.2018;102(12):1615-20.

23. Arita R, Fukuoka S, Morishige N. Functional morphology of the lipid layer of the tear film. Cornea. 2017;36(Suppl 1):S60-S6.

24. Akpek EK, Klimava A, Thorne JE, Martin D, Lekhanont K, OstrovskyA. Evaluation of patients with dry eye for presence of underlying Sjögren syndrome. Cornea. 2009;28(5):493-7.

25. Huang J, Hindman HB, Rolland JP. In vivo thickness dynamics measurement of tear film lipid and aqueous layers 
with optical coherence tomography and maximum-likelihood estimation. Opt Lett. 2016;41(9):1981-4.

26. Nelson JD, Wright JC. Conjunctival goblet cell densities in ocular surface disease. Arch Ophthalmol. 1984;102(7):1049-51.

27. Aranha dos Santos V, Schmetterer L, Groschl M, Garhofer G, Schmidl D, Kucera M, et al. In vivo tear film thickness measurement and tear film dynamics visualization using spectral domain optical coherence tomography. Opt Express. 2015;23(16):21043-63.

28. BaiY,Ngo W, GuB,ZhangY, NicholsJJ.Animaging systemintegrating optical coherence tomography and interferometry for in vivo measurement of the thickness and dynamics of the tear film. BioMed Eng OnLine. 2018;17(1):164.

29. Wang J, Aquavella J, Palakuru J, Chung S. Repeated measurements of dynamic tear distribution on the ocular surface after instillation of artificial tears. Invest Ophthalmol Vis Sci. 2006;47(8):3325-9.

30. Wozniak PA, Schmidl D, Bata AM, Fondi K, Witkowska KJ, Aranha dos Santos V, et al. Effect of different lubricant eye gels on tear film thickness as measured with ultrahigh-resolution optical coherence tomography. Acta Ophthalmol. 2017;95(4):e307-e13.

31. Schmidl D, Werkmeister R, Kaya S, Unterhuber A, Witkowska KJ, Baumgartner R, et al. A controlled, randomized double-blind study to evaluate the safety and efficacy of chitosan- $\mathrm{N}$-acetylcysteine for the treatment of dry eye syndrome. JOcul Pharmacol Ther. 2017;33(5):375-82.

32. Kaya S, Schmidl D, Schmetterer L, Witkowska KJ, Unterhuber A, Aranha dos Santos V, et al. Effect of hyaluronic acid on tear film thickness as assessed with ultra-high resolution optical coherence tomography. Acta Ophthalmol. 2015;93(5):439-43.

33. Schmidl D, Witkowska KJ, Kaya S, Baar C, Faatz H, Nepp J, et al. The association between subjective and objective parameters for the assessment of dry-eye syndrome. Invest Ophthalmol Vis Sci. 2015;56(3):1467-72.

34. Szegedi S, Scheschy U, Schmidl D, Aranha Dos Santos V, Stegmann H, Adzhemian N, et al. Effect of single instillation of two hyaluronic acid-based topical lubricants on tear film thickness in patients with dry eye syndrome. J Ocul PharmacolTher. 2018;34(9):605-11.

35. Schmidl D, Schmetterer L, Witkowska KJ, Unterhuber A, Aranha dos Santos V, Kaya S, et al. Tear film thickness after treatment with artificial tears in patients with moderate dry eye disease. Cornea. 2015;34(4):421-6.

36. Schmidl D, Bata AM, Szegedi S, Aranha dos Santos V, Stegmann $\mathrm{H}$, Fondi K, et al. Influence of perfluorohexyloctane eye drops on tear film thickness in patients with mild to moderate dry eye disease: a randomized controlled clinical trial.J Ocul Pharmacol Ther. 2020;36(3):154-61.

37. Kallab M, Szegedi S, Hommer N, Stegmann H, Kaya S, Werkmeister RM, et al. Topical low dose preservative-free hydrocortisone reduces signs and symptoms in patients with chronic dry eye: a randomized clinical trial. Adv Ther. 2020;37(1):329-41.

38. Aranha dos Santos V, Schmetterer L, Triggs GJ, Leitgeb RA, Groschl M, Messner A, et al. Super-resolved thickness maps of thin film phantoms and in vivo visualization of tear film lipid layer using OCT. Biomed Opt Express. 2016;7(7):2650-70.

39. StegmannH,Aranha dos Santos V, MessnerA, UnterhuberA, Schmidl D, Garhöfer G, et al. Automatic assessment of tear film and tear meniscus parameters in healthy subjects using ultrahigh-resolution optical coherence tomography. Biomed OptExpress. 2019;10(6):2744-56.
40. Napoli PE, Nioi M, Mangoni L, Gentile P, Braghiroli M, d'Aloja E, et al. Fourier-domain OCT imaging of the ocular surface and tear film dynamics: a review of the state of the art and an integrative model of the tear behavior during the inter-blinkperiod and visual fixation. J Clin Med. 2020;9(3):668.

41. Shen M, LiJ, WangJ, MaH, CaiC, TaoA, etal. Upper andlower tear menisci in the diagnosis of dry eye. Invest Ophthamol Vis Sci. 2009;50(6):2722-6.

42. Holly FJ. Physical chemistry of the normal and disordered tear film. Trans OphthalmolSoc U K. 1985;104(Pt4):374-80.

43. Schmidl D, Schlatter A, Chua J, Tan B, Garhöfer G, Schmetterer L. Novel approaches for imaging-based diagnosis of ocular surface disease. Diagnostics (Basel). 2020;10(8):589.

44. Wang J, Cui L, Shen M, Perez VL, Wang MR. Ultra-high resolution optical coherence tomography for monitoring tear meniscus volume in dry eye after topical cyclosporine treatment. Clin Ophthalmol. 2012;6:933-8.

45. Imamura H, Tabuchi H, Nakakura S, Nagasato D, Baba H, Kiuchi Y. Usability and reproducibility of tear meniscus values generated via swept-source optical coherence tomography and the slit lamp with a graticule method. Int Ophthalmol.2018;38(2):679-86.

46. Tittler EH, Bujak MC, Nguyen P, Zhang X, Li Y, Yiu SC, et al. Between-grader repeatability of tear meniscus measurements using Fourier-domain OCT in patients with dry eye. Ophthalmic Surg Lasers Imaging. 2011;42(5):423-7.

47. Tukenmez-Dikmen N, Yildiz EH, Imamoglu S, Turan-Vural E, Sevim MS. Correlation of dry eye workshop dry eye severity grading system with tear meniscus measurement by optical coherence tomography and tear osmolarity. Eye Contact Lens. 2016;42(3):153-7.

48. Nguyen P, Huang D, Li Y, Sadda SR, Ramos S, Pappuru RR, et al. Correlation between optical coherence tomography-derived assessments of lower tear meniscus parameters and clinical features of dry eye disease. Cornea. 2012;31(6):680-5.

49. Altan-Yaycioglu R, Sizmaz S, Canan H, Coban-Karatas M. Optical coherence tomography for measuring the tear film meniscus: correlation with schirmer test and tear-film breakup time. Curr Eye Res. 2013;38(7):736-42.

50. Chen Q, ZhangX, Cui L, HuangQ, Chen W, MaH, etal. Upper and lower tear menisci in Sjögren's syndrome dry eye. Invest Ophthalmol Vis Sci. 2011;52(13):9373-8.

51. QiuX, Gong L, Lu Y, Jin H, Robitaille M. The diagnostic significance of Fourier-domain optical coherence tomography in Sjögren syndrome, aqueous tear deficiency and lipid tear deficiencypatients.ActaOphthalmol.2012;90(5):e359-e66.

52. Carracedo G, Pastrana C, Serramito M, Rodriguez-Pomar C. Evaluation of tear meniscus by optical coherence tomography after different sodium hyaluronate eyedrops instillation. Acta Ophthalmol.2019;97(2):e162-e9.

53. Wang Y, Zhuang H, Xu J, Wang X, Jiang C, Sun X. Dynamic changes in the lower tear meniscus after instillation of artificial tears. Cornea. 2010;29(4):404-8.

54. BujakMC, YiuS,ZhangX,LiY, HuangD. Serialmeasurement of tear meniscus by FD-OCT after instillation of artificial tears in patients with dry eyes. Ophthalmic Surg Lasers Imaging. 2011;42(4):308-13.

55. Yoo Y-S, Na K-S, Byun Y-S, Shin JG, Lee BH, Yoon G, et al. Examination of gland dropout detected on infrared meibography by using optical coherence tomography meibography. Ocul Surf. 2017;15(1):130-138.e1.

56. Ju MJ, ShinJG, HoshiS, Yasuno Y, LeeBH, Tang S, etal. Threedimensional volumetric human meibomian gland investigation using polarization-sensitive optical coherence tomography. JBiomed Opt. 2014;19(3):30503. 
57. Cui X, Wu Q, Zhai Z, Yang Y, Wei A, Xu J, et al. Comparison of the meibomian gland openings by optical coherence tomography in obstructive meibomian gland dysfunction and normal patients. JClin Med. 2020;9(10):E3181.

58. Hwang HS, Shin JG, Lee BH, Eom TJ, Joo C-K. In vivo 3D meibography of the human eyelid using real time imaging Fourier-domain OCT. PLoS ONE. 2013;8(6):e67143.

59. Napoli PE, Coronella F, Satta GM, Iovino C, Sanna R, Fossarello M. A simple novel technique of infrared meibography by means of spectral-domain optical coherence tomography: a cross-sectional clinical study. PLoS ONE. 2016;11(10):e165558.

60. Veres A, Tapasztó B, Kosina-Hagyó K, Somfai GM, Németh J. Imaging lid-parallel conjunctival folds with OCT and comparing its grading with the slit lamp classification in dry eye patients and normal subjects. Invest Ophthamol Vis Sci. 2011;52(6):2945-51.

61. Tapasztó B, Veres A, Kosina-Hagyó K, Somfai GM, Németh J. OCTimagingoflid-parallel conjunctivalfoldsinsoftcontact lens wearers. Optom Vis Sci. 2011;88(10):1206-13.

62. Bandlitz S, Purslow C, Murphy PJ, Pult H. Influence of conjunctival folds on calculated tear meniscus volume along the lower eyelid. Ocul Surf. 2016;14(3):377-84.

63. Pult $\mathrm{H}$, Riede-Pult BH. Impact of conjunctival folds on central tear meniscus height. Invest Ophthamol Vis Sci. 2015;56(3):1459-66.

64. Doh SH, Kim EC, Chung SY, Kim MS, Chung SK, Shin MC, et al. Optical coherence tomography imaging of human lacrimal glands: an in vivo study. Ophthalmology. 2015;122(11):2364-6.

65. Sung Y, Park JS, Lew H. Measurement of lacrimal punctum using spectralis domain anterior optical coherence tomography. Acta Ophthalmol.2017;95(7):e619-e24.

66. Timlin HM, Keane PA, Day AC, Salam T, Abdullah M, Rose GE, et al. Characterizing the lacrimal punctal region usinganteriorsegmentopticalcoherencetomography.Acta Ophthalmol.2016;94(2):154-9.

67. Allam RSHM, Ahmed RA. Evaluation of the lower punctum parameters and morphology using spectral domain anterior segment optical coherence tomography. J Ophthalmol. 2015; https://doi.org/10.1155/2015/591845.
68. Kamal S, Ali MJ, Ali MH, Naik MN. Fourier domain optical coherence tomography with $3 \mathrm{D}$ and en face imaging of the punctum and vertical canaliculus: a step toward establishing a normative database. Ophthal Plast Reconstr Surg. 2016;32(3):170-3.

69. Wawrzynski JR, Smith J, Sharma A, Saleh GM. Optical coherence tomography imaging of the proximal lacrimal system. Orbit. 2014;33(6):428-32.

70. Kashani AH, Chen C-L, Gahm JK, Zheng F, Richter GM, Rosenfeld PJ, et al. Optical coherence tomography angiography: a comprehensive review of current methods and clinical applications. Prog Retin Eye Res. 2017;60:66-100.

71. SpaideRF, FujimotoJG, Waheed NK, SaddaSR, StaurenghiG. Optical coherence tomography angiography.Prog RetinEye Res. 2018;64:1-55.

72. Hau SC, Devarajan K, Ang M. Anterior segment optical coherence tomography angiography and optical coherence tomography in the evaluation of episcleritis and scleritis. Ocul Immunol Inflamm. 2019; https://doi.org/10.1080/ 09273948.2019.1682617.

73. Pichi F, Roberts P, Neri P. The broad spectrum of application of optical coherence tomography angiography to the anteriorsegment of the eyein inflammatory conditions: a review of the literature. J Ophthalmic Inflamm Infect. 2019;9(1):18.

74. Fung SSM, Stewart RMK, Dhallu SK, Sim DA, Keane PA, Wilkins MR, et al. Anterior segment optical coherence tomographic angiography assessment of acute chemical injury. Am J Ophthalmol. 2019;205:165-74.

75. Efron N. Grading scales for contact lens complications. Ophthalmic PhysiolOpt. 1998;18(2):182-6.

76. Akagi T, Uji A, Okamoto Y, Suda K, Kameda T, Nakanishi H, et al. Anterior segment optical coherence tomography angiography imaging of conjunctiva and intrasclera in treated primary open-angle glaucoma. Am J Ophthalmol. 2019;208:313-22.

Hinweis des Verlags Der Verlag bleibt in Hinblick auf geografische Zuordnungen und Gebietsbezeichnungen in veröffentlichten Karten und Institutsadressen neutral. 